\title{
Thrombotic risk in gastroenteropancreatic neuroendocrine tumor patients: a single-center experience
}

\author{
Sara Massironi ${ }^{\mathrm{a}, \mathrm{b}}$, Federica Cavalcolic, Andrea Artonid, Valentina Sciola ${ }^{\mathrm{e}}$, Alessandra Zillie, \\ Clorinda Ciafardinie, Roberta Elisa Rossi ${ }^{\mathrm{f}, \mathrm{g}}$
}

San Gerardo Hospital and University of Milano-Bicocca, Monza; Istituti Clinici Zucchi, Monza; Fondazione IRCCS Ca' Granda - Ospedale Policlinico, Milan; Fondazione IRCCS Istituto Nazionale dei Tumori, Milan; Università degli Studi di Milano, Italy

\section{Abstract}

${ }^{\mathrm{a} D}$ Division of Gastroenterology - European Reference Network on Hepatological Diseases (ERN RARE-LIVER) San Gerardo Hospital (Sara Massironi); ' University of Milano-Bicocca, Monza (Sara Massironi); 'Gastroenterology and Endoscopy Unit, Istituti Clinici Zucchi, Monza (Federica Cavalcoli); d“Bianchi Bonomi Hemophilia and Thrombosis Center", Fondazione IRCCS Ca' Granda - Ospedale Policlinico di Milano (Andrea Artoni); ${ }^{\mathrm{e}}$ Gastroenterology and Endoscopy Unit, Fondazione IRCCS Ca' Granda Ospedale Maggiore Policlinico di Milano (Valentina Sciola, Alessandra Zilli, Clorinda Ciafardini); ${ }^{\mathrm{f}} \mathrm{HPB}$ Surgery, Hepatology and Liver Transplantation Unit, Fondazione IRCCS Istituto Nazionale dei Tumori, Milan (Roberta Elisa Rossi); ${ }^{g}$ Department of Pathophysiology and Transplantation, Università degli Studi di Milano, Milan, Italy (Roberta Elisa Rossi)

\section{Conflict of Interest: None}

Correspondence to: Sara Massironi, MD PhD, Division of Gastroenterology - San Gerardo Hospital, via Pergolesi 33, 20900 Monza (MB)

e-mail: sara.massironi@libero.it

Received 7 September 2020; accepted 28 December 2020; published online 26 February 2021

DOI: https://doi.org/10.20524/aog.2021.0613

\section{Introduction}

Gastroenteropancreatic (GEP) neuroendocrine neoplasms (NENs) comprise a heterogeneous group of neoplasms that have peculiar and specific characteristics, often different from other neoplasms. Venous thromboembolism (VTE), which comprises deep vein thrombosis (DVT) and pulmonary embolism (PE), is a frequent complication in cancer patients especially in the GEP area [1]. Among the gastrointestinal neoplasms, pancreatic cancer (e.g., pancreatic ductal adenocarcinoma) is well-known to carry an elevated thrombotic risk, with a 1-year cumulative incidence of 6-16\%, dependent on cancer stage and treatment [2]. Whether or not NENs have the same risk of VTE as do the other GEP cancers, has not been systematically evaluated yet.

Cancer patients have a several-fold increased risk of venous thrombosis compared with the general population or patients without cancer, with relative risks ranging from 4-7 [1]. VTE is associated with increased morbidity and mortality and hinders its clinical management. The mechanisms underlying the association between VTE and cancer are far from being clearly 
understood. Firstly, it has been reported that the expression of tissue factor on tumor cells and the pro-thrombotic properties of mucins contribute to the thrombotic risk in malignancy. Furthermore, there is evidence that tissue factor expression, which may result from proto-oncogene expression and tumorsuppressor gene inhibition, confers a pro-angiogenic state, which may enhance the aggressiveness and invasiveness of cancer [2].

VTE is a complex multifactorial disease, which involves the interaction between acquired or inherited predispositions to thrombosis and various risk factors. Even if VTE occurrence has been described as associated with neoplastic disease, and some independent risk factors and predictors for incidental and recurrent VTE have been identified, no specific data are available in the literature in the specific setting of NENs, except for functioning glucagon-secreting neoplasms. Glucagonoma is a very rare functioning pancreatic tumor and its incidence rate is approximately 2.4/100,000,000 [3,4]. Glucagonoma may clinically manifest with the so-called " $4 \mathrm{D}$ syndrome" i.e., diabetes, dermatitis, DVT, and depression [5]. Even in this setting, the mechanism for coagulopathy is poorly understood [6]. Furthermore, only recently, a single study assessed the incidence of VTE in patients with abdominal NENs who underwent surgery from 2008-2015 [7], suggesting that routinely extending VTE prophylaxis after surgery may be necessary for pancreatic NENs, whilst it is probably unnecessary for all the NENs patients [7]. The present study aimed to assess the actual incidence of venous thromboembolism in a cohort of GEP-NEN patients at a single referral center.

\section{Patients and methods}

\section{Study design}

This study is a retrospective, single-center cohort study conducted in the Academic Hospital of Fondazione IRCCS Ca' Granda Ospedale Maggiore Policlinico, Milan, Italy. The retrospective analysis of a database of patients prospectively collected at a single tertiary referral center was performed. Demographic, clinical, surgical, and pathological data were collected and analyzed. All the patients or their legal representatives provided written informed consent for the anonymous review of their data for research purposes. This retrospective study was approved by the local Ethics Committee.

The primary endpoint of this study was the assessment of the incidence of VTE in a series of GEP-NEN patients. The secondary aim was the investigation of risk factors for VTE.

\section{Study population}

From January 2000 to December 2016 all the patients diagnosed with functional and non-functional GEP-NENs at Fondazione IRCCS Ca' Granda Ospedale Maggiore
Policlinico, Milan, Italy were retrospectively evaluated. Data were collected from medical records. All the patients were asked to complete a risk factors questionnaire and the history of thrombosis was taken. The Khorana score [8] was calculated for each patient. A minimum 3-month follow-up was required. Inclusion criteria were: age $>18$ years, histologically confirmed GEP-NENs, availability of minimal histological parameters (classification according to WHO, Ki-67) [9], availability of clinical parameters (TNM staging), availability of instrumental morphologic parameters (ultrasound/ computed tomography [CT]/ magnetic resonance imaging/ nuclear medicine), availability of biochemical data (chromogranin A, 5-Hydroxyindoleacetic acid, vasoactive intestinal peptide, glucagon, somatostatin, and gastrin). The exclusion criteria were the presence or previous history of other malignancies and pre-existing thrombophilic conditions (i.e., factor $\mathrm{V}$ Leiden mutation, prothrombin mutation, antithrombin III deficiency, protein $\mathrm{C}$ deficiency, protein $\mathrm{S}$ deficiency, and antiphospholipid syndrome).

Each tumor was classified according to the site of origin, presence/absence of clinical syndrome, WHO2010 classification based on Ki-67 index, identifying well-differentiated grade (G) 1 and 2 neuroendocrine tumors (NETs G1 and G2), and poorly differentiated G3 neuroendocrine carcinomas [9], and presence/absence of nodal or distant metastases (staged according to the TNM staging system) $[10,11]$.

\section{Index events}

DVT and PE were considered major thrombotic events. Lower-limb compression ultrasonography and/or pulmonary angiography CT were performed in all the cases of suspected DVT and PE, respectively. The primary outcome was the composite of symptomatic or incidentally diagnosed distal or proximal lower extremity DVT or PE objectively confirmed by imaging tests. Incidental (unsuspected) VTE was defined as VTE detected on imaging tests performed for other reasons, mainly for staging the disease. Major VTE was considered tumor-related if it occurred within 1 year before the GEP-NEN diagnosis, being 10\% the risk prevalence of undiagnosed cancer in patients with unprovoked VTE at 12 months [12].

\section{Statistical analysis}

Baseline data were summarized using standard descriptive statistics. Categorical variables were expressed as number (percentage). The results of continuous variables were presented as medians and ranges or interquartile ranges. All the data were tested for normal distribution by the KolmogorovSmirnov test. Any differences between percentages were evaluated using Fisher's exact test. Differences between groups were evaluated by means of the Mann-Whitney, and Kruskal-Wallis tests, followed by Dunn's multiple comparison test whenever appropriate. The survival rate and the overall 
cumulative incidence of VTE were measured using the KaplanMeier method. A P-value $<0.05$ was considered statistically significant. The analyses were performed by software using GraphPad Prism Version 5.00 for Windows (GraphPad Software, San Diego, California, USA).

\section{Results}

During the study period, 160 patients (72 male, median age 68 years) with GEP-NEN were included in the study; the median follow-up was 62 (range 8-196) months. The site of the primary tumor was the gut in 99 patients, the pancreas in 55, and unknown in 7. The tumor grading was G1 in 93 patients, G2 in 35, G3 in 4, and not available in 27. The TNM stage was: I in 76 patients, II in 17, III in 23, and IV in 44.

Among them, during a median follow-up duration of 62 months, 12 patients (7.5\%) (8 males and 4 females, median age 64 [range 54-71] years) developed DVT or PE (Fig. 1), corresponding to an estimated 6, 12 and 24 months overall cumulative incidence of $3.3,4.7$ and $6.8 \%$ (Fig. 2). The clinical

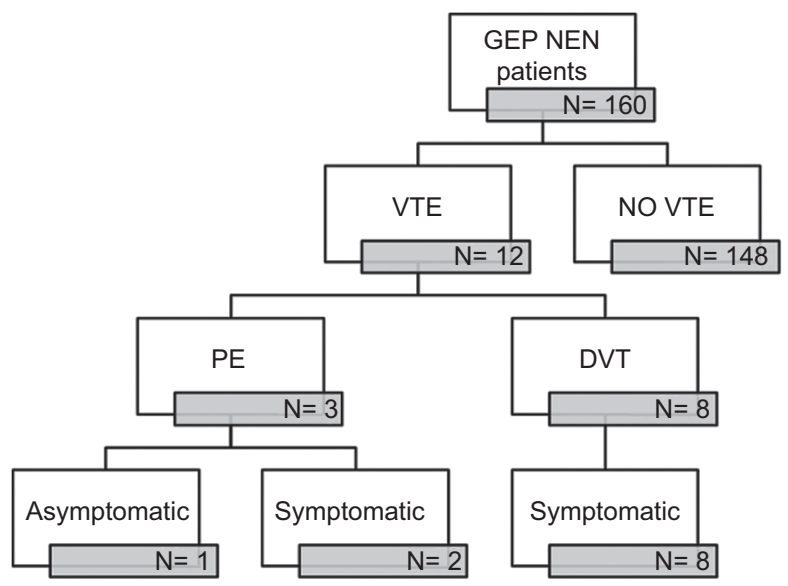

Figure 1 Flowchart of the patients enrolled in the study VTE, venous thromboembolism; GEP NEN, gastroenteropancreatic neuroendocrine neoplasm; PE, pulmonary embolism; DVT, deep vein thrombosis

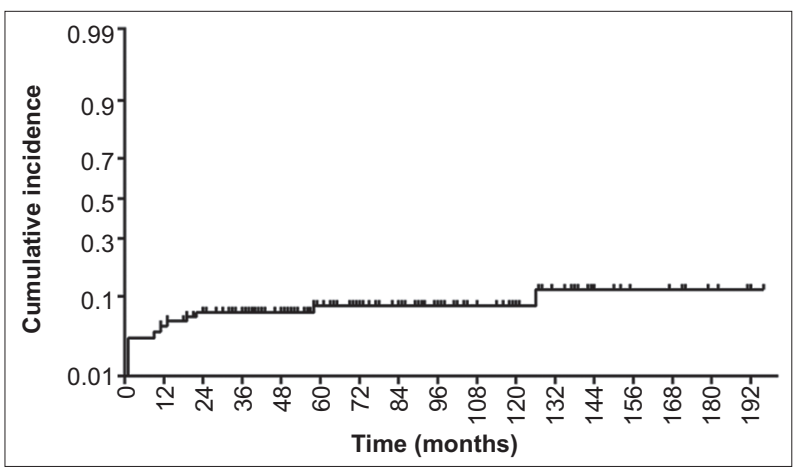

Figure 2 Risk of deep vein thrombosis or pulmonary embolism in patients with gastroenteropancreatic neuroendocrine neoplasms in the study period features of the 12 patients who developed VTE in comparison to the whole cohort of 160 GEP-NEN patients are provided in Table 1.

Nine patients (75\%) had DVT, symptomatic in all cases. The main symptoms at presentation were unilateral inferior limb swelling (67\%), cramping pain (56\%), and cutaneous dyschromia (33\%). Three patients (25\%) had PE, symptomatic in 2 cases and asymptomatic in 1 , and 1 patient was diagnosed with concomitant PE and DVT. In 9 patients the primary tumor was located in the pancreas $(75 \%)$, in 2 cases in the gut $(17 \%)$, and in the remaining one, the primary tumor site remained unknown. Two patients had a functioning tumor (gastrinoma in one, glucagon-secreting neoplasm in the other). The tumor grading was G1 in 3 patients, G2 in 6, G3 in 2 cases, and not available in one case. Five patients were at stage IV of the disease, 2 at stage III, 3 at stage II, and 2 at stage I. In 3 cases VTE developed after surgery for GEP-NEN, and no cases of VTE for surgery unrelated to GEP-NEN were observed. In 10 patients VTE developed after or concurrently the GEPNEN diagnosis (median 12 months), whilst in 2 patients VTE developed 4 and 6 months before diagnosis, respectively. Seven patients underwent surgical intervention: in 2 cases an $\mathrm{R} 0$ resection was obtained, whereas in the other 5 patients surgery was not curative and the patients received further therapy (namely, somatostatin analogs (SSAs), chemotherapy, and radio-targeted therapy (PRRT)]. The 5 patients who were not eligible for surgery received SSAs alone or in combination with chemotherapy, PRRT, or targeted therapies (everolimus, sunitinib). In 3 patients VTE developed during GEP-NEN therapy, in 2 during SSA therapy, and in 1 during SSA plus everolimus therapy.

Regarding other risk factors besides surgery, 1 patient had a previous DVT, and another one had atrial fibrillation. All the patients presented with no significant alteration in the platelet count, hemoglobin, or coagulation tests and did not have bone fractures, trauma, or were on estroprogestinic therapy. None of the patients had a Khorana score $>2$, so that none was considered at "high risk" according to this score. All the patients developing VTE received anticoagulant treatment for at least 6 months after VTE. During anticoagulant therapy, one patient had gastrointestinal bleeding requiring blood transfusion.

The rate of pancreatic localization was significantly higher in the cases of GEP-NEN with VTE than those without VTE (75\% vs. $34 \%, \mathrm{P}=0.002$, Fisher's exact test) (Fig. 3). Moreover, a significantly higher proportion of G2-G3 neoplasms vs. G1 ones was observed in NENs with VTE compared to the proportion of G2-G3 in the whole cohort of NEN patients ( $67 \%$ vs. $39 \%$, $\mathrm{P}=0.006$, Fisher's exact test) (Fig. 4). No significant statistical difference was observed with regard to age, sex, functioning status, and stage distribution, even if there was a trend to higher disease staging in GEP-NENs with VTE.

Two of $12(16.6 \%)$ patients died during the study period (for one patient death was related to PE), similar to the death rate for the whole cohort of 160 GEP-NENs patients (20 deaths among 160 patients, $12.5 \%$ ). The overall survival was similar in the VTE subgroup compared to the one observed in the entire study population (log-rank test $\mathrm{P}=0.12$ ). 
Table 1 Characteristics of GEP-NEN vs. GEP-NEN with VTE patients

\begin{tabular}{|c|c|c|c|}
\hline Characteristics & GEP-NEN patients $(\mathrm{N}=160)$ & GEP-NEN patients with VTE $(\mathrm{N}=11)$ & P-value \\
\hline Age, median (range) & $68(18-83)$ & $64(54-71)$ & n.s. \\
\hline Male/female, $\mathrm{N}$ (\% of male) & $72 / 88(45)$ & $8 / 3(73)$ & n.s. \\
\hline BMI $\left(\mathrm{kg} / \mathrm{m}^{2}\right)$ (median, IQR) & $24.6(22.1-27.3)$ & $24.5(21.5-27.6)$ & n.s. \\
\hline Platelet count $\left(10^{9} / \mathrm{L}\right)$, median (IQR) & $220(175-259)$ & $232(147-308)$ & n.s. \\
\hline Leukocytes count $\left(10^{9} / \mathrm{L}\right)$, median (IQR) & $5.8(4.6-8.3)$ & $6.5(3.9-8.6)$ & n.s. \\
\hline Hemoglobin (mmol/L), median (IQR) & $12(10.8-13.8)$ & $13.7(11.1-14.9)$ & n.s. \\
\hline Functioning/non- functioning, n (\%) & $44 / 116(38)$ & $2 / 9(22)$ & n.s. \\
\hline $\begin{array}{l}\text { Site, N (\%) } \\
\text { Pancreas } \\
\text { Gut } \\
\text { Unknown }\end{array}$ & $\begin{array}{c}55(34) \\
99(62) \\
7(4)\end{array}$ & $\begin{array}{c}8(73) \\
2(18) \\
1(9)\end{array}$ & 0.002 \\
\hline $\begin{array}{l}\text { Staging } \\
\text { I } \\
\text { II } \\
\text { III } \\
\text { IV }\end{array}$ & $\begin{array}{l}76(48) \\
17(11) \\
23(14) \\
44(27)\end{array}$ & $\begin{array}{l}2(18) \\
3(27) \\
2(18) \\
4(37)\end{array}$ & n.s. \\
\hline $\begin{array}{l}\text { Grading } \\
\text { G1 } \\
\text { G2/G3 } \\
\text { n.a. }\end{array}$ & $\begin{array}{l}93(58) \\
39(24) \\
27(17)\end{array}$ & $\begin{array}{c}3(27) \\
7(64) \\
1(9)\end{array}$ & 0.006 \\
\hline
\end{tabular}

VTE, venous thromboembolism; GEP-NEN, gastroenteropancreatic neuroendocrine neoplasm; n.s., not significant; $n . a$., not available; IQR, interquartile range; BMI, body mass index

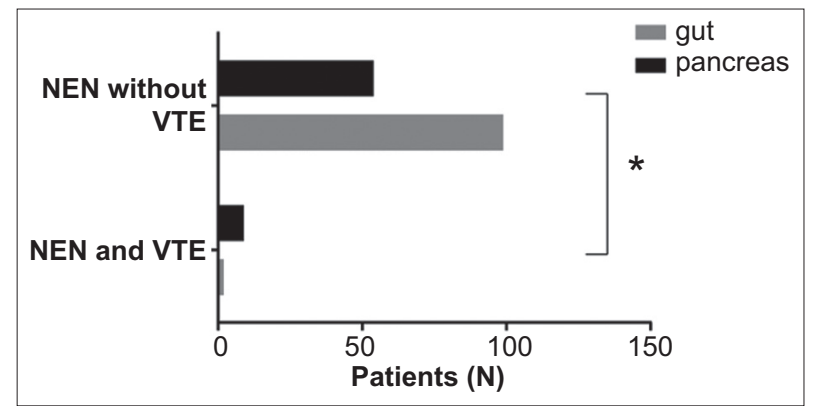

Figure 3 Distribution of the patients according to the primary tumor site (pancreas vs. gut) in the neuroendocrine neoplasm (NEN) patients with venous thromboembolism (VTE) and in all the NEN patients $(\mathrm{P}=0.002$, Fisher's exact test $)$

\section{Discussion}

According to the present study, patients with GEP-NENs, either functioning or non-functioning, harbor a considerable risk of venous thrombosis, which seems to be higher than the average population risk [13-16]. The risk of thrombosis seems particularly high in the case of pancreatic NEN, in patients with G2 or G3 neoplasms, and in those at an advanced tumor stage. However, the clear mechanism underlying the association between GEP-NENs and the increased risk of developing VTE is still unclear, also taking into account the huge heterogeneity which represents a hallmark for all types of NENs.

The association between cancer and thrombosis is well established. Several mechanisms may explain why cancer

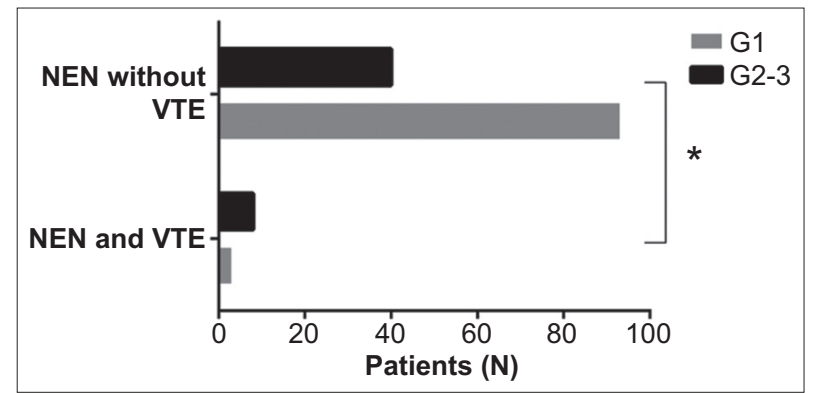

Figure 4 Distribution of the patients according to the grade of the primary tumor (G1 vs. G2-G3) in the neuroendocrine neoplasm (NEN) patients with venous thromboembolism (VTE) and all the NEN patients $(\mathrm{P}=0.006$, Fisher's exact test $)$

increases the propensity to develop VTE. Besides the hypercoagulable status, which usually characterizes neoplastic patients, malignancy itself, surgery, indwelling intravenous catheters, chemo-radiotherapy, and intercurrent medical complications, such as infection, all contribute to the increased risk of thrombosis in cancer patients [2]. Several tumor sites, including the pancreas, stomach, lungs, brain, ovaries, and kidneys, and also lymphoma have the strongest reported association with thrombosis development $[17,18]$.

In the present series, the risk of thrombosis appears particularly high for pancreatic NEN patients and those with higher Gs. This is in line with the data reporting on the exocrine counterpart, as pancreatic cancer is one of the tumors with the highest association with VTE (approximately 20 events per 100 patient-years). Moreover, patients with high grade and an 
advanced tumor stage are more likely to continue receiving medications for their disease, so one can speculate that each drug class such as SSAs, chemotherapy, PRRT, or target therapy may play a role. However, due to the small number of index events (DVT and PE) in the current series, it was not possible to carry out more structured and solid statistical analyses.. Further studies with larger samples are needed to draw more solid conclusions.

No specific risk factors as platelet/coagulation alterations, estroprogestinic therapy, trauma, or prolonged immobilization have been observed in our patients besides the presence of NEN and surgery in 3 patients. Of note, the risk of VTE appears to be independent of hospitalization as 3 patients only (25\%) in our present series developed VTE in the perioperative phase. Moreover, none of the patients had a Khorana score $>2$, so that none was considered at "high risk" according to this score. One patient had a previous DVT, treated with lowweight heparin for 6 months, 3 years before NEN diagnosis and another patient had atrial fibrillation and was on warfarin treatment when VTE occurred.

Concerning the interval between GEP-NEN diagnosis and VTE occurrence, we have observed the higher incidence of VTE in the first 2 years from the tumor diagnosis, as reported in the literature for all types and stages of cancer [19-21].

It has been reported that the thromboembolism rate was 4-13 times higher among the cases with metastatic disease than those with localized disease [19,22]. In line with these findings, a trend for higher risk of developing VTE has been observed, in the present series, for those patients at a more advanced disease stage. However, statistically significant results were not achieved in the present series, probably because of the relatively small number of events observed in the cohort of patients considered.

According to some previous studies, the development of VTE has been a significant predictor of death within 1 year for all cancer types analyzed [19-21,23-26]. In the current series, 2 of $12(16.6 \%)$ patients died during the study period and for one patient the death was related to PE, similar to the death rate for the whole cohort of 160 GEP-NENs patients (20 deaths among 160 patients, $12.5 \%$ ). A possible explanation may be the different and heterogeneous natural history of GEP-NENs compared to the exocrine counterpart.

All the patients developing VTE received anticoagulation for at least 6 months after the diagnosis of VTE, even if no specific treatment protocols are available in this setting. According to the available guidelines on VTE associated with cancer, the initial treatment should be carried out with lowweight heparin for 6 months, if tolerated [27]. Warfarin and other new oral anticoagulants are acceptable alternatives if heparin is impractical and anticoagulation is indicated $[28,29]$. In the presence of active malignancy, the guidelines suggest carrying on with the anti-coagulation, also taking into account the individual patient's status and any bleeding risk [2]. This prophylaxis has been shown to be effective for myeloma, pancreatic ductal adenocarcinoma but in absolute numbers, these cancers lead to a few venous thromboembolic events. At present, VTE risk scores cannot be used as a discriminatory criterion to select a high-risk population that could really benefit from this prevention, as we observed in the present study. In this series, 3 patients continued their anticoagulation therapy for a longer period: 7 and 2 years for 2 patients, respectively, and long-term therapy for the patient with concomitant atrial fibrillation.

The strength of the present study comes from the current lack of data in the literature regarding this association, which makes this study among the first to focus on the risk of VTE in the neuroendocrine setting. One main limitation of the study is its retrospective nature which reduces the strength of our data. However, taking into consideration the low incidence of VTE in the general population and the lack of reported data in NEN we designed a retrospective study to assess the incidence of VTE. The promising data observed could hopefully represent the epidemiological starting point for further prospective studies. Other limitations are the single-center design of the study and the small sample size, which might have affected some results. Further studies with external validation and larger series are necessary to confirm these results.

Finally, the incidence percentages herein reported possibly underestimate the true incidence of VTE, as only clinically evident events with a clear-cut diagnosis have been considered, some subclinical events having possibly been missed out. In particular, it is possible that DVT or PE in this series of GEP-NEN could have been missed in asymptomatic patients, as in this study population the vast majority of the VTE cases were clinically symptomatic. Therefore, the real incidence of VTE could be even higher than that reported in this paper.

In summary, although the intrinsic limitations of the current study do not allow us to draw solid conclusions, it appears that patients with GEP-NEN, either functioning or non-functioning, might harbor a considerable risk of VTE. The risk of thrombosis seems particularly high for male patients, in the case of pancreatic NENs, in patients with G2 or G3 neoplasms, and in those at an advanced tumor stage. Physicians dealing with GEP-NEN patients should be aware of this risk, keeping in mind that these tumors are very heterogeneous in terms of biological behavior. Also, in the case of adjunctive risk factors, such as surgery, trauma, immobilization, adequate antithrombotic prophylaxis, and preventive measures against VTE might be encouraged. Further prospective studies with larger samples are required in order to understand the biological mechanisms causing VTE in patients with GEPNENs and thus to select specific subgroups of NEN patients who need adequate antithrombotic prophylaxis and preventive measures.

\section{Acknowledgment}

We thank Marcello Hinxman-Allegri for the language editing (grammatical assistance, stylistic suggestions). 


\section{Summary Box}

\section{What is already known:}

- Venous thromboembolism is a frequent complication in cancer patients

- In pancreatic neuroendocrine neoplasms (NENs) extended venous thromboembolism prophylaxis after surgery has been suggested for the increased risk of thrombotic events

- Glucagonoma clinical syndrome is characterized by a hypercoagulable state, diabetes, dermatitis, and depression

\section{What the new finding is:}

- Patients with gastroenteropancreatic NENs have an increased risk of venous thrombosis, particularly higher in those with pancreatic NEN, G2 or G3 neoplasms, and with an advanced tumor stage

\section{References}

1. Timp JF, Braekkan SK, Versteeg HH, Cannegieter SC. Epidemiology of cancer-associated venous thrombosis. Blood 2013;122:17121723.

2. Watson HG, Keeling DM, Laffan M, Tait RC, Makris M, Haematology BCfSi. Guideline on aspects of cancer-related venous thrombosis. Br J Haematol 2015;170:640-648.

3. Yao JC, Eisner MP, Leary C, et al. Population-based study of islet cell carcinoma. Ann Surg Oncol 2007;14:3492-3500.

4. Öberg K. Management of functional neuroendocrine tumors of the pancreas. Gland Surg 2018;7:20-27.

5. Eldor R, Glaser B, Fraenkel M, Doviner V, Salmon A, Gross DJ. Glucagonoma and the glucagonoma syndrome - cumulative experience with an elusive endocrine tumour. Clin Endocrinol (Oxf) 2011;74:593-598.

6. Grozinsky-Glasberg S, Shimon I, Korbonits M, Grossman AB. Somatostatin analogues in the control of neuroendocrine tumours: efficacy and mechanisms. Endocr Relat Cancer 2008;15:701-720.

7. Skertich NJ, Gerard J, Poirier J, et al. Do all abdominal neuroendocrine tumors require extended postoperative VTE prophylaxis? A NSQIP analysis. J Gastrointest Surg 2019;23:788-793.

8. Khorana AA, Kuderer NM, Culakova E, Lyman GH, Francis CW. Development and validation of a predictive model for chemotherapy-associated thrombosis. Blood 2008;111:4902-4907.

9. Rindi G, Arnold R, Bosman FT. Nomenclature and classification of neuroendocrine neoplasms of the digestive system. In: Bosman FT, Carneiro F, Hruban RH, Theise ND (editors): WHO classification of tumors of the digestive system. International Agency for Research on Cancer (IRAC): Lyon; 2010, pp. 13-14.

10. Rindi G, Klöppel G, Alhman H, et al. TNM staging of foregut (neuro)endocrine tumors: a consensus proposal including a grading system. Virchows Arch 2006;449:395-401.

11. Rindi G, Klöppel G, Couvelard A, et al. TNM staging of midgut and hindgut (neuro) endocrine tumors: a consensus proposal including a grading system. Virchows Arch 2007;451:757-762.

12. Carrier M, Le Gal G, Wells PS, Fergusson D, Ramsay T, Rodger MA. Systematic review: the Trousseau syndrome revisited: should we screen extensively for cancer in patients with venous thromboembolism? Ann Intern Med 2008;149:323-333.

13. Cushman M, Tsai AW, White RH, et al. Deep vein thrombosis and pulmonary embolism in two cohorts: the longitudinal investigation of thromboembolism etiology. Am J Med 2004;117:19-25.

14. Nordström M, Lindblad B, Bergqvist D, Kjellström T. A prospective study of the incidence of deep-vein thrombosis within a defined urban population. J Intern Med 1992;232:155-160.

15. Oger E. Incidence of venous thromboembolism: a communitybased study in Western France. EPI-GETBP Study Group. Groupe d'Etude de la Thrombose de Bretagne Occidentale. Thromb Haemost 2000;83:657-660.

16. Naess IA, Christiansen SC, Romundstad P, Cannegieter SC, Rosendaal FR, Hammerstrøm J. Incidence and mortality of venous thrombosis: a population-based study. J Thromb Haemost 2007;5:692-699.

17. Agnelli G. Venous thromboembolism and cancer: a two-way clinical association. Thromb Haemost 1997;78:117-120.

18. Baron JA, Gridley G, Weiderpass E, Nyrén O, Linet M. Venous thromboembolism and cancer. Lancet 1998;351:1077-1080.

19. Chew HK, Wun T, Harvey D, Zhou H, White RH. Incidence of venous thromboembolism and its effect on survival among patients with common cancers. Arch Intern Med 2006;166:458-464.

20. Sørensen HT, Mellemkjaer L, Olsen JH, Baron JA. Prognosis of cancers associated with venous thromboembolism. $N$ Engl J Med 2000;343:1846-1850.

21. Rickles FR, Levine MN. Epidemiology of thrombosis in cancer. Acta Haematol 2001;106:6-12

22. Wun T, White RH. Epidemiology of cancer-related venous thromboembolism. Best Pract Res Clin Haematol 2009;22:9-23.

23. Levitan N, Dowlati A, Remick SC, et al. Rates of initial and recurrent thromboembolic disease among patients with malignancy versus those without malignancy. Risk analysis using Medicare claims data. Medicine (Baltimore) 1999;78:285-291.

24. Palareti G, Legnani C, Lee A, et al. A comparison of the safety and efficacy of oral anticoagulation for the treatment of venous thromboembolic disease in patients with or without malignancy. Thromb Haemost 2000;84:805-810.

25. Trujillo-Santos J, Prandoni P, Rivron-Guillot K, et al. Clinical outcome in patients with venous thromboembolism and hidden cancer: findings from the RIETE Registry. $J$ Thromb Haemost 2008;6:251-255.

26. Prandoni P, Lensing AW, Piccioli A, et al. Recurrent venous thromboembolism and bleeding complications during anticoagulant treatment in patients with cancer and venous thrombosis. Blood 2002;100:3484-3488.

27. Lee AY, Levine MN, Baker RI, et al. Low-molecular-weight heparin versus a coumarin for the prevention of recurrent venous thromboembolism in patients with cancer. $N$ Engl J Med 2003;349:146-153.

28. van der Hulle T, Huisman MV, Klok FA. Meta-analysis of the efficacy and safety of new oral anticoagulants in patients with cancer-associated acute venous thromboembolism: reply. J Thromb Haemost 2014;12:2138-3219.

29. Zhi-Chun G, Yi-Dan Y, Sheng-Yan Y, et al. Direct versus conventional anticoagulants for treatment of cancer-associated thrombosis: a pooled and interaction analysis between observational studies and randomized clinical trials. Ann Transl Med 2020;8:95. 